\title{
REDUCING SAMPLING ERROR BY PROLATE SPHEROIDAL WAVE FUNC- TIONS AND FRACTIONAL FOURIER TRANSFORM
}

\author{
Jian-Jiun Ding, Soo-Chang Pei \\ Department of Electrical Engineering, National Taiwan University, Taipei, Taiwan, R.O.C \\ Email address: pei@cc.ee.ntu.edu.tw
}

\begin{abstract}
It is known that one can use Shannon's theory to sample a bandlimited signal. In this paper, we introduce how to use prolate spheroidal wave functions (PSWFs) to sample a time-limited and nearly band-limited signal. PSWFs have the property of optimal energy concentration. Thus we can apply it for sampling theory to reduce the aliasing error of the recovered signal. We derive the theory that can estimate the upper bound of the error. With it, we can determine that, to achieve certain accuracy, how many samples we should acquire. Moreover, we combine the proposed sampling theory with the fractional Fourier transform (FRFT). We also find an important theory, i.e., to achieve a certain degree of accuracy, the number of sampling points required for a signal is proportional to the 'area' of its time-frequency distribution.
\end{abstract}

\section{INTRODUCTION}

From Shannon's theory, if $g(t)$ is a bandlimited signal:

$G(\omega)=0 \quad$ when $\omega>\Omega, \quad$ where $G(\omega)=F T[g(t)]$, we can sample $g(t)$ with the interval of $\pi / \Omega$ :

$$
g_{\mathrm{s}}[n]=g(n \pi / \Omega), \quad n=\ldots,-1,0,1,2,3, \ldots .
$$

(2)

To recover $g(t)$ from $g_{s}[n]$, we can pass it through a lowpass filter with cutoff $\Omega$ or, equivalently:

$$
\hat{g}(t)=\sum_{n=-\infty}^{\infty} g_{s}[n] \frac{\sin (\Omega t-n \pi)}{\Omega t-n \pi} .
$$

We can recover $g(t)$ from $g_{\mathrm{s}}[n]$ perfectly (i.e., $\left.g(t)=\hat{g}(t)\right)$ if $g(t)$ is bandlimited. However, in nature, most of the signals are not bandlimited. Instead, most of them are time-limited:

$$
g(t)=0 \quad \text { when } t \notin[-T, T] \text {. }
$$

Since $g(t)$ is time-limited, it is impossible for $g(t)$ to be bandlimited. It is only possible that $g(t)$ is nearly bandlimited:

$$
\frac{\int_{\Omega}^{\infty}|G(\omega)|^{2} d \omega+\int_{-\infty}^{\Omega}|G(\omega)|^{2} d \omega}{\int_{-\infty}^{\infty}|G(\omega)|^{2} d \omega}=\varepsilon
$$

where $\varepsilon$ is a small value near to zero (however, $\varepsilon$ is never equal to zero). Thus, if we using (3) to recover $g(t)$, 'aliasing effect' will occur and we can not recover the original signal perfectly. The error between the recovered signal $\hat{g}(t)$ and $g(t)$ is:

$$
\frac{\int_{-\infty}^{\infty}|\hat{g}(t)-g(t)|^{2} d t}{\int_{-\infty}^{\infty}|g(t)|^{2} d t}=\frac{\int_{-\infty}^{\infty}|\hat{G}(\omega)-G(\omega)|^{2} d \omega}{\int_{-\infty}^{\infty}|G(\omega)|^{2} d \omega}=\varepsilon+\varepsilon_{1}
$$

where $\hat{G}(\omega)=\operatorname{FT}[\hat{g}(t)]$ and $\varepsilon_{1}$ is the aliasing error:

$$
\varepsilon_{1}=\frac{\int_{\Omega}^{\Omega}|\hat{G}(\omega)-G(\omega)|^{2} d \omega}{\int_{-\infty}^{\infty}|G(\omega)|^{2} d \omega}=\frac{\int_{\Omega}^{\Omega}\left|\sum_{m, m \neq 0} G(\omega+2 m \Omega)^{2}\right| d \omega}{\int_{-\infty}^{\infty}|G(\omega)|^{2} d \omega},
$$

Thus the sampling error comes from two places: (a) $\varepsilon$, the high frequency component of $G(\omega)$. It is removed by the lowpass filter in (3). (b) $\varepsilon_{1}$ : Due to aliasing error, the low frequency components of $G(\omega)$ are interfered by the high frequency component.

In this paper, we use prolate spheroidal wave functions (PSWFs) together with the fractional Fourier transform (FRFT) to improve sampling theory. PSWFs are the continuous functions satisfying the following homogeneous integral equation:

$$
\begin{gathered}
\int_{-T}^{T} \frac{\sin \left[\Omega\left(t_{1}-t\right)\right]}{\pi\left(t_{1}-t\right)} \psi_{n, T, \Omega}(t) d t=\lambda_{n, T, \Omega} \psi_{n, T, \Omega}\left(t_{1}\right), \\
1>\lambda_{0, T, \Omega}>\lambda_{1, T, \Omega}>\lambda_{2, T, \Omega}>\lambda_{3, T, \Omega}>\ldots \ldots . .>0 .
\end{gathered}
$$

(We will simplify the notations of PSWFs and their eigenvalues as $\psi_{n}(t)$ and $\lambda_{n}(t)$, respectively). They are orthogonal and complete on both the interval of $[-T, T]$ and the interval of $(-\infty, \infty)$ :

$$
\int_{-T}^{T} \psi_{m}(t) \psi_{n}(t) d t=\lambda_{n} \delta_{m, n}, \quad \int_{-\infty}^{\infty} \psi_{m}(t) \psi_{n}(t) d t=\delta_{m, n} .
$$

They are useful for analyzing the properties of the finite-interval Fourier transform (fi-FT):

$$
\begin{aligned}
& O_{f i-F}[f(t)]=F_{1}(\omega)=(2 \pi)^{-1 / 2} \int_{-T}^{T} e^{-j \omega t} f(t) d t, \\
& t \in[-T, T], \quad \omega \in[-\Omega, \Omega] .
\end{aligned}
$$

The power preservation ratio of the fi-FT can be calculated from:

$$
\text { energy prsv. ratio } R=\frac{\int_{-\Omega}^{\Omega}\left|F_{1}(\omega)\right|^{2} d \omega}{\int_{-T}^{T}|f(t)|^{2} d t}=\frac{\sum_{n=0}^{\infty}\left|a_{n}\right|^{2} \lambda_{n}^{2}}{\sum_{n=0}^{\infty}\left|a_{n}\right|^{2} \lambda_{n}} \text {. }
$$

where $f(t)=\sum_{n=0}^{\infty} a_{n} \psi_{n}(t)$.

Notice that when $f(t)=a_{0} \psi_{0}(t), R=\lambda_{0}$. In this case, the energy preservation ratio is maximal. In addition, among the functions orthogonal to $\psi_{0}(t), \psi_{1}(t)$ is the one that has the largest energy preservation ratio $R$. Among the functions orthogonal to $\psi_{0}(t)$ and $\psi_{1}(t), \psi_{2}(t)$ is the one that has the largest value of $R$, and so on. Thus PSWFs have the properties of optimal energy preservation when both the time and the frequency extensions are finite. It is helpful for optics analysis, laser, filter design, etc. [2][3].

In addition to the above applications, PSWFs are also helpful for improving sampling theory. This application was first discussed in [4]. In this paper, we improve their works. First, we derive the upper bound of the error of the recovered signal. With 
it, we can estimate, to achieve a certain degree of accuracy, how many sample values we should acquire. In addition, in [4], the sampling points should be uniform. For our algorithms, the locations of sampling points can be arbitrary. The only constraint is that the samples of PSWFs at these points should construct a full-rank matrix.

Moreover, we will combine the theory of PSWFs with the fractional Fourier transform (FRFT). The FRFT is more generalized than the FT. It can make the time-frequency analysis more flexible. We find an important theory that the 'interval-width product' of the time-frequency distribution of a signal determines the number of sampling points. Due to the theory, we can vary the parameter $\alpha$ of the FRFT such that the product is minimal. Then we can use the least number of samples to achieve a certain degree of accuracy (or use the same number of samples to achieve higher accuracy).

\section{SAMPLING AND RECONSTRUCTION BY PROLATE SPHEROIDAL WAVE FUNCTIONS}

For a time-limited signal in (4), suppose that the ratio of the energy outside the interval of $\omega \in[-\Omega, \Omega]$ is $\varepsilon$, as in (5). We can use the following algorithm to sample and reconstruct it by prolate spheroidal wave functions (PSWFs):

(1) First, we project $g(t)$ on $\operatorname{Span}\left\{\psi_{0}(t), \psi_{1}(t), \psi_{2}(t), \ldots \psi_{N-1}(t)\right\}$ :

$$
g(t) \rightarrow g_{N}(t) \quad \text { where } g_{N}(t)=\sum_{n=0}^{N-1} a_{n} \psi_{n}(t) .
$$

(2) Then we choose $N$ sampling points $t_{1}, t_{2}, t_{3}, \ldots \ldots, t_{N}$ among the intervals of $t \in[-T, T]$. The locations of $t_{n}$ 's can be chosen arbitrary. They are unnecessary to be uniform. The only requirement is $\operatorname{det}(\mathbf{A}) \neq 0$ where

$$
\mathbf{A}=\left[\begin{array}{cccc}
\psi_{0}\left(t_{1}\right) & \psi_{0}\left(t_{2}\right) & \cdots & \psi_{0}\left(t_{N}\right) \\
\psi_{1}\left(t_{1}\right) & \psi_{1}\left(t_{2}\right) & \cdots & \psi_{1}\left(t_{N}\right) \\
\vdots & \vdots & \ddots & \vdots \\
\psi_{N-1}\left(t_{1}\right) & \psi_{N-1}\left(t_{2}\right) & \cdots & \psi_{N-1}\left(t_{N}\right)
\end{array}\right]
$$

(3) After the sampling points are chosen, we obtain $N$ sampling values: $g_{N}\left(t_{1}\right), g_{N}\left(t_{2}\right), g_{N}\left(t_{3}\right)$, $g_{N}\left(t_{N}\right)$.

To reconstruct the original signal from the sampling values,

(1) First, recover $a_{n}$ 's from:

$$
\mathbf{a}=\mathbf{g}_{\mathbf{N}} \mathbf{A}^{-1}
$$

where $\mathbf{a}=\left[a_{0}, a_{1}, a_{2}, \ldots \ldots, a_{N-1}\right]$,

$$
\mathbf{g}_{\mathbf{N}}=\left[g_{N}\left(t_{1}\right), g_{N}\left(t_{2}\right), g_{N}\left(t_{3}\right), \ldots \ldots \ldots \ldots g_{N}\left(t_{N}\right)\right] .
$$

(2) Then the original signal can be recovered from

$$
g(t) \approx g_{N}(t)=\sum_{n=0}^{N-1} a_{n} \psi_{n}(t)
$$

We then introduce an important theorem

[Theorem 1] When using PSWFs to sample a time-limited signal with $N$ sampling points, the upper bound of the error of the recovered signal is

$$
E<\frac{\varepsilon}{1-\lambda_{N}} \text {. }
$$

where $\varepsilon$ is defined in (5) and $\lambda_{N}$ is the $N^{\text {th }}$ eigenvalue of the integral equation in (8).

(Proof) The FT of a PSWF is:

$$
\psi_{n}(t) \stackrel{F T}{\longrightarrow} \mu_{n}(\omega)=(-j)^{n} \sqrt{\frac{T \lambda_{n}}{\Omega}} \psi_{n}\left(\frac{T}{\Omega} \omega\right) .
$$

Thus, if $G(\omega)$ and $G_{N}(\omega)$ are the FT of $g(t)$ and $g_{N}(t)$, then

$$
G(\omega)=\sum_{n=0}^{\infty} a_{n} \mu_{n}(\omega), \quad G_{N}(\omega)=\sum_{n=0}^{N-1} a_{n} \mu_{n}(\omega) \text {. }
$$

From Parseval's theory,

$$
\begin{aligned}
& \int_{-\infty}^{\infty}\left|g(t)-g_{N}(t)\right|^{2} d t=\int_{-\infty}^{\infty}\left|G(\omega)-G_{N}(\omega)\right|^{2} d \omega, \\
& =\int_{-\infty}^{\infty} \sum_{n=N}^{\infty}\left|a_{n}\right|^{2}\left|\mu_{n}(\omega)\right|^{2} d \omega, \\
& \int_{-\infty}^{\infty}|g(t)|^{2} d t=\int_{-\infty}^{\infty} \sum_{n=0}^{\infty}\left|a_{n}\right|^{2}\left|\mu_{n}(\omega)\right|^{2} d \omega .
\end{aligned}
$$

From (10) and (21),

$$
\begin{aligned}
& \int_{-\infty}^{\infty}\left|\mu_{n}(\omega)\right|^{2} d \omega=\int_{-\infty}^{\infty}\left|(-j)^{n} \sqrt{\frac{T \lambda_{n}}{\Omega}} \psi_{n}\left(\frac{T}{\Omega} \omega\right)\right|^{2} d \omega \\
& =\lambda_{n} \int_{-\infty}^{\infty}\left|\psi_{n}(\omega)\right|^{2} d \omega=\lambda_{n}
\end{aligned}
$$

Therefore, the normalized difference between $g_{N}(t)$ and $g(t)$ is:

$$
E=\frac{\int_{-\infty}^{\infty}\left|g(t)-g_{N}(t)\right|^{2} d t}{\int_{-\infty}^{\infty}|g(t)|^{2} d t}=\frac{\sum_{n=N}^{\infty}\left|a_{n}\right|^{2} \lambda_{n}}{\sum_{n=0}^{\infty}\left|a_{n}\right|^{2} \lambda_{n}}=\frac{\sum_{n=N}^{\infty} b_{n}}{\sum_{n=0}^{\infty} b_{n}} .
$$

where $b_{n}=\left|a_{n}\right|^{2} \lambda_{n}$. To find the lower bound of $E$, from (5),

$$
\begin{aligned}
& \int_{-\Omega}^{\Omega}|G(\omega)|^{2} d \omega=(1-\varepsilon) \int_{-\infty}^{\infty}|G(\omega)|^{2} d \omega, \\
& \int_{-\Omega}^{\Omega} \sum_{n=0}^{\infty}\left|a_{n}\right|^{2}\left|\mu_{n}(\omega)\right|^{2} d \omega=(1-\varepsilon) \sum_{n=0}^{\infty} b_{n} .
\end{aligned}
$$

Then, from (10) and (21),

$$
\int_{-\Omega}^{\Omega}\left|\mu_{n}(\omega)\right|^{2} d \omega=\lambda_{n} \int_{-T}^{T}\left|\psi_{n}(t)\right|^{2} d t=\lambda_{n}^{2} \text {. }
$$

Therefore, (28) is changed into

$$
\sum_{n=0}^{\infty} b_{n} \lambda_{n}=(1-\varepsilon) \sum_{n=0}^{\infty} b_{n}, \quad \sum_{n=0}^{\infty} b_{n}\left(1-\lambda_{n}\right)=\varepsilon \sum_{n=0}^{\infty} b_{n} .
$$

From the fact that $\lambda_{n}<\lambda_{N}$ if $n>N$,

$$
\begin{aligned}
& \sum_{n=N}^{\infty} b_{n}\left(1-\lambda_{N}\right)<\sum_{n=N}^{\infty} b_{n}\left(1-\lambda_{n}\right)<\sum_{n=0}^{\infty} b_{n}\left(1-\lambda_{n}\right), . \\
& \sum_{n=N}^{\infty} b_{n}\left(1-\lambda_{N}\right)<\varepsilon \sum_{n=0}^{\infty} b_{n} .
\end{aligned}
$$

After substituting it into (26), we obtain (20)

\#

In (20), the error upper bound is determined by $\varepsilon$ and $\lambda_{N}$. Moreover, the values of $\lambda_{n}$ is determined by the 'interval-width product' of the signal [1]:

$$
P=2 T \times 2 \Omega=4 T \Omega \text {. }
$$

The value of $\lambda_{n}$ grows with $P$. For a certain value of $P$,

$$
\begin{array}{ll}
\lambda_{n}>0.99 & \text { when } 0 \leq n<P / 2 \pi-\Delta, \\
0.01<\lambda_{n}<0.99 & \text { when } P / 2 \pi-\Delta \leq n \leq P / 2 \pi+\Delta, \\
\lambda_{n}<0.01 & \text { when } n>P / 2 \pi+\Delta
\end{array}
$$

where $\Delta$ is a small value no more than 5 . Thus, the recovered error of the sampling by PSWFs is dominated by three factors: 
(1) $\varepsilon$ : how much energy is outside the interval of $\omega \in[\Omega, \Omega]$.

(2) $N$ : the number of sampling points.

(3) $P=4 T \Omega$ : the 'interval-width product' of the signal.

Eq. (20) gives us a way to choose the strategy for sampling a signal. If we want the error to be no more than $E_{M}$, we can adjust two parameters, $N$ and $\Omega$ (both $\varepsilon$ and $P$ can be controlled by $\Omega$ ) to make $\varepsilon /\left(1-\lambda_{N}\right)$ no more than $E_{M}$.

If $N$ is sufficient large, then, from (33), $\lambda_{N} \approx 0$. In this case, the upper bound of error is

$$
\varepsilon /\left(1-\lambda_{N}\right) \approx \varepsilon
$$

Comparing it with (6), we find that, when using the original algorithm based on Shannon's theory, the error is $\varepsilon+\varepsilon_{1}$. However, when we use PSWFs for sampling, the error is reduced to $\varepsilon$. The aliasing effect will not appear when using PSWFs for sampling. Thus using PSWFs can improve the accuracy of sampling.
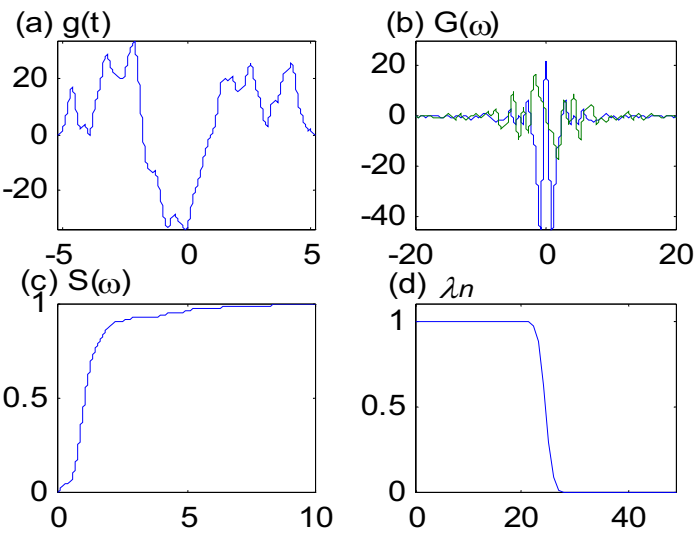

(e) by original algorithm

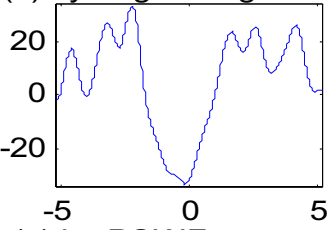

(g) by PSWFs
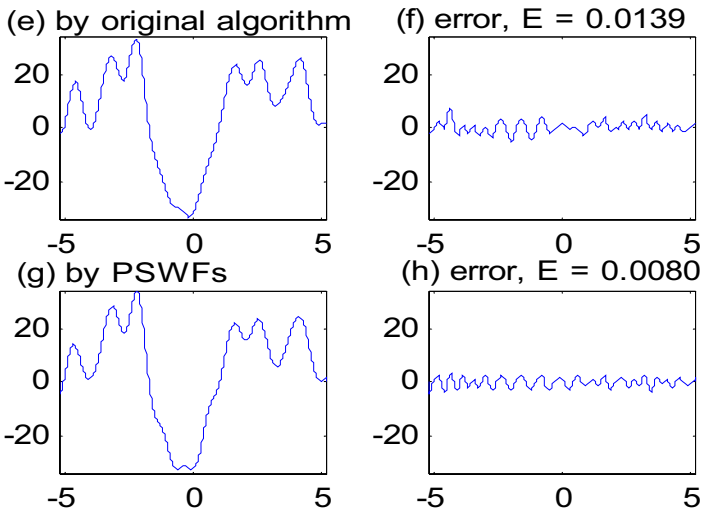

Fig. 1 The experiment that use PSWFs to sample a time-limited and nearly band-limited signal $g(t)$. (c) The energy ratio, defined in (36), (d) the eigenvalues of PSWFs, (e)(f) the recovered signal and the error when using Shannon's theorem, $(\mathrm{g})(\mathrm{h})$ the recovered signal and the error when using PSWFs for sampling.

In Fig. 1, we give an example. Here we use a time-limited signal. The signal in Fig. 1(a) is time limited:

$g(t)=0 \quad$ for $t \notin[-5.2,5.2]$.

We show the FT of $g(t)$ in Fig. 1(b). Although $g(t)$ is not bandlimited, however, most of the energy of $G(\omega)$ is concentrated on the low frequency region. In Fig. 2(c), we plot $S(\omega)$ where

$$
S(\omega)=\frac{\int_{-\omega}^{\omega}|G(\tau)|^{2} d \tau}{\int_{-\infty}^{\infty}|G(\tau)|^{2} d \tau} .
$$

Since

$\mathrm{S}(7.5)=0.9914$

only $0.86 \%$ of the energy is outside of the region of $\omega \in[-7.5$, 7.5]. Therefore, we then use (8) to find the PSWFs where $T=5.2$ and $\Omega=7.5$. The eigenvalues of the PSWFs we obtain are plotted in Fig. 1(d). We choose $N=26$, i.e., the number of sampling points is 26 . Since

$\lambda_{25}=0.2919$,

from (20), the upper bound of the error is

$E=0.01215$.

Notice that it is the 'upper bound'. In fact, the error can be further less. In Fig. 1(g), we use the algorithm we propose to recover the original signal. The difference between the recovered signal and the original one is plotted in Fig. 1(f) and the error is $E=0.0080$.

We then compare it with the case where we use the original Shannon's theorem to sample and reconstruct the signal. The recovered signal is plotted in Fig. 1(e) and the error is

$E=0.0139$.

Thus using the PSWFs can reduced the sampling error.

We then describe two important theories.

[Theorem 2] If, in (20), the upper bound of error ratio (denoted by $\left.E_{M}, E_{M}=\varepsilon /\left(1-\lambda_{N}\right)\right)$ is fixed, the number of sampling points $N$ is dependent on $\Omega$. The optimal case (i.e., $N$ is minimal) is achieved when

$0.99>\lambda_{N}>0.01$.

Due to the constraint of paper length, we don't prove it here. It can be proved from Theorem 1 and (33).

[Theorem 3] If the upper bound of error ratio $E_{M}$ is fixed, the number of sampling points required for a time-limited signal is

$$
N=\frac{R}{2 \pi}+\tau
$$

where $R=4 T B$, the extension of the signal is $t \in[-T, T]$, and $B$ is the frequency threshold such that the energy ratio of the signal outside the region of $\omega \in[-B, B])$ is less than $E_{M}$ :

$$
\frac{\int_{-B}^{B}|g(t)|^{2} d t}{\int_{-\infty}^{\infty}|g(t)|^{2} d t}>1-E_{M}
$$

In the case where $g(t)$ is not time-limited but $|g(t)|$ is very small when $|t|$ is large, the inequality in (44) is changed into

$$
\left(1-\frac{\int_{-T}^{T}|g(t)|^{2} d t}{\int_{-\infty}^{\infty}|g(t)|^{2} d t}\right)+\left(1-\frac{\int_{-\Omega}^{\Omega}|G(\omega)|^{2} d \omega}{\int_{-\infty}^{\infty}|G(\omega)|^{2} d \omega}\right)<E_{M} .
$$

In addition, in (43), $\tau$ is a small value dependent on the signal $g(t)$. In usual, $|\tau|<<R / 2 \pi$ and

$$
N \approx R / 2 \pi \text {. }
$$

In other word, the number of sampling points is dominated by the interval-width product $R$, i.e., the product of the widths of the time interval and the frequency interval.

(Proof) Since $E_{M}=\varepsilon /\left(1-\lambda_{N}\right), \varepsilon=\left(1-\lambda_{N}\right) E_{M}$, which is smaller than $E_{M}$. Thus, in (5), the cutoff frequency $\Omega$ should be larger than $B$ where $B$ satisfies (44). Thus, from (33) and Theorem 2

$$
N>2 T \Omega / \pi-5>2 T B / \pi-5=R / 2 \pi-5
$$

This is the lower bound of $N$. In addition, for the case where $\lambda_{N}=$ $0.01, \varepsilon=0.99 E_{M}$. In this condition, $\Omega=B+\sigma$ where $\sigma$ is a small positive value. From (33), 
$N<2 T \Omega / \pi+5=R / 2 \pi+2 T \sigma / \pi+5$

The value of $N$ in the optimal case should be smaller than the value of $N$ calculated from $\lambda_{N}=0.01$. Therefore,

$R / 2 \pi-5<N<R / 2 \pi+2 T \sigma / \pi+5$,

and $N$ is near to $R / 2 \pi$.

\section{FURTHER IMPROVEMENT BY FRACTIONAL FOURIER TRANSFORMS}

The fractional Fourier transform (FRFT) is defined as [5]:

$$
G_{\alpha}(u)=\left(\frac{1-j \cot \alpha}{2 \pi}\right)^{1 / 2} \int_{-\infty}^{\infty} e^{\frac{j}{2} u^{2} \cot \alpha-j u t \csc \alpha+\frac{j}{2} t^{2} \cot \alpha} g(t) d t
$$

The FRFT can rotate the Wigner distribution (WDF) of a signal by angle $\alpha$ in the time-frequency plane [6]. For example, in Fig. 2, the WDF of $g(t)$ is plotted in Fig. 2(b). After doing the FRFT with $\alpha=0.71$, the WDF of $G_{\alpha}(u)$ is plotted in Fig. 2(c), which is just a rotation of Fig. 2(b) by angle 0.71 .

In previous section, we found that we can use PSWFs to improve sampling theory, and the number of sampling points is determined by the 'interval-width product' $R$. In fact, the theory can be further improved by the FRFT. For example, suppose that the upper bound of sampling error is $E_{x}$ is 0.01 . From Fig. 2(b), the energy of $g(t)$ are in the region of:

$$
t \in[-8.3,8.3], \quad \omega \in[-8.6,8.6] \text {. }
$$

In this case, the time frequency product $R$ is 285.52 and, from (43), the number of sampling points should be $N \approx 285.52 / 2 / \pi \approx$ 45.442. However, after doing the FRFT, in Fig. 2(c), we find that the energy of $G_{\alpha}(u)$ is concentrated on the following region:

$$
u \in[-7.21,7.21], \quad \omega \in[-7.52,7.52] \text {. }
$$

The time-frequency product now is reduced into $R=4 * 7.21 *$ $7.52=216.88$ and the number of sampling points is reduced into $N \approx R / 2 \pi=34.51$. Thus, with the FRFT, we can use fewer sampling points to achieve the same degree of accuracy (or, alternatively, using the same number of sampling points to achieve higher accuracy).

We also do the experiments that use 36 points to sample the signal $g(t)$ in Fig. 2(a) by

(I) the original sampling theorem,

(II) the proposed sampling algorithm in Sec. 2,

(III) the proposed sampling theorem together with the FRFT, and calculation the recovered errors. The results are:
(I) $E=0.01373$,
(II) $E=0.01163$,

(III) $E=0.006997$.

Thus using the PSWFs together with the FRFT can further reduce the sampling errors.

For a signal $g(t)$, we can vary $\alpha$ iteratively to minimize the interval-width product $R_{\alpha}$ and do the optimal sampling where

$$
\begin{gathered}
R_{\alpha}=4 T_{\alpha} \Omega_{\alpha} \quad \text { and } T_{\alpha} \text { and } \Omega_{\alpha} \text { satisfy } \\
\left(1-\frac{\int_{-T_{\alpha}}^{T_{\alpha}}\left|G_{\alpha}(u)\right|^{2} d u}{\int_{-\infty}^{\infty}\left|G_{\alpha}(u)\right|^{2} d u}\right)+\left(1-\frac{\int_{\Omega_{\alpha}}^{\Omega_{\alpha}}\left|G_{\alpha+\frac{\pi}{2}}(u)\right|^{2} d u}{\int_{-\infty}^{\infty}\left|G_{\alpha+\frac{\pi}{2}}(u)\right|^{2} d u}\right)<E_{M} .
\end{gathered}
$$

In fact, the minimal value of $R_{\alpha}$ is determined by the 'area' of the time-frequency distribution of a signal. Therefore,
[Theorem 4] When using PSWFs together with the FRFT, if the bound of error ratio $E_{M}$ is fixed, the number of sampling points required for a signal is approximated to

$$
N=\frac{A}{2 \pi}+\tau
$$

where $A=\min \left(R_{\alpha}\right)$ is near to the 'area' of the time-frequency distribution of a signal.

(a) $\mathrm{g}(\mathrm{t})$

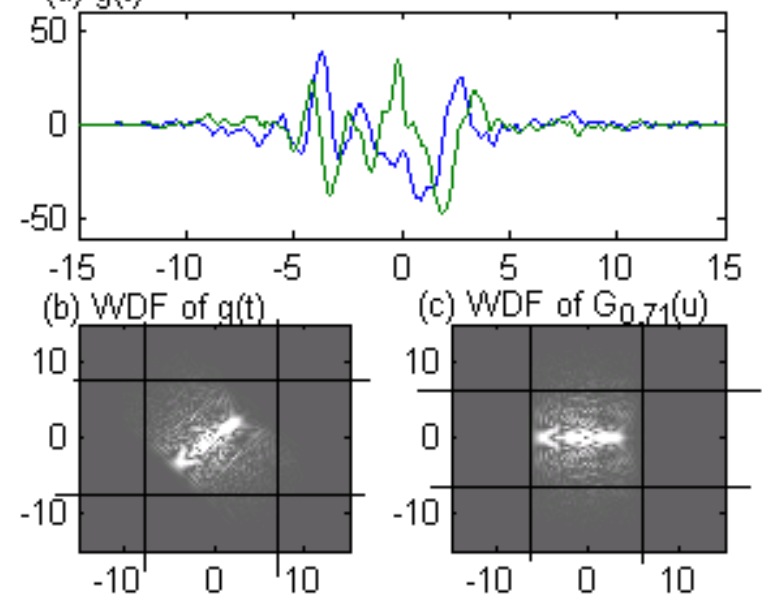

Fig. $2 g(t)$ and the WDF of $g(t)$ and $G_{\alpha}(u)$ where $G_{\alpha}(u)$ is the FRFT of $g(t)$.

\section{CONCLUSIONS}

We have improved sampling theory by PSWFs and the FRFT. With them, the accuracy of the recovered signal can be much improved when using the same number of samples. We also derive several important theories, including the upper bound of the error and that the number of sampling points for a signal is determined by the area of its time-frequency distribution.

\section{REFERENCES}

[1] D. Slepian and H. O. Pollak, 'Prolate spheroidal wave functions, Fourier analysis and uncertainty-I,'Bell Syst. Tech. J., vol. 40, pp. 43-63, 1961.

[2] A. Papoulis and M. S. Bertran, "Digital filtering and prolate functions," IEEE Trans. Circuit Theory, vol. 19, pp. 674-681.

[3] B. R. Frieden, "Ch. 8: Evaluation, design and extrapolation methods for optical signals, based on use of the prolate functions," in Progress in Optics, E. Wolf Ed., v. 9, pp. 311-407.

[4] G. G. Walter and X. A. Shen, "Sampling with prolate spheroidal wave functions", Sampling Theory in Signal and Image Processing, vol. 2, pp. 25-52, Jan. 2003.

[5] H. M. Ozaktas, Z. Zalevsky, and M. A. Kutay, "The Fractional Fourier Transform with Applications in Optics and Signal Processing", John Wiley \& Sons, 2000.

[6] A. W. Lohmann, "Image rotation, Wigner rotation, and the fractional Fourier transform," J. Opt. Soc. Am. A, vol. 10, no. 10, pp. 2181-2186, Oct. 1993.

[7] J. L. Brown, "Bounds for truncation error in sampling expansions of band-limited signals", IEEE Trans. Inf. Theory, vol. 15, no. 4, pp. 440-444, July 1969. 